\title{
Towards photon radiotherapy treatment planning with high Z nanoparticle radiosensitisation agents: the Relative Biological Effective Dose (RBED) framework
}

\author{
Jeremy M. C. Brown ${ }^{1,2,3^{*}}$, Gerard G. Hanna ${ }^{4}$, Nathanael Lampe ${ }^{5}$, Balder Villagomez-Bernabe', James R. Nicol ${ }^{6}$, \\ Jonathan A. Coulter ${ }^{6}$ and Fred J. Currell ${ }^{1}$
}

\section{*Correspondence: \\ jeremy.brown@cern.ch \\ ${ }^{1}$ School of Mathematics and Physics, Queen's University Belfast, Belfast, Northern Ireland, UK \\ Full list of author information is available at the end of the article}

\begin{abstract}
A novel treatment planning framework, the Relative Biological Effective Dose (RBED), for high Z nanoparticle (NP)-enhanced photon radiotherapy is developed and tested in silico for the medical exemplar of neoadjuvant (preoperative) breast cancer MV photon radiotherapy. Two different treatment scenarios, conventional and high Z NP enhanced, were explored with a custom Geant4 application that was developed to emulate the administration of a single 2 Gy fraction as part of a 50 Gy radiotherapy treatment plan. It was illustrated that there was less than a $1 \%$ difference in the dose deposition throughout the standard and high Z NP-doped adult female phantom. Application of the RBED framework found that the extent of possible biological response with high Z NP doping was great than expected via the dose deposition alone. It is anticipated that this framework will assist the scientific community in future high Z NP-enhanced in-silico, pre-clinical and clinical trials.
\end{abstract}

Keywords: Radiotherapy, Nanoparticles, Treatment planning, Radiosensitisers, Biological effect modelling, Theranostic nanoparticles

\section{Introduction}

Photon radiotherapy is one of the most commonly employed approaches in the treatment of cancer (Delaney et al. 2005; Urruticoechea et al. 2010). Since its first application in the 1920s, photon radiotherapy has undergone continuous refinement through the development of new technologies and increased understanding of radiation biology (Mayles et al. 2007; Joiner and van der Kogel 2009). Further optimisation has included the combination of radiotherapy and systemic therapy with resultant improvements in both local control and overall survival (Aupérin et al. 2010). Over the last decade, there has been increasing interest in the use of nanomedicines both as single-agent anti-cancer therapeutics and in combination with radiotherapy (Chen et al. 2016; Schuemann et al. 2016; Marples and Dhar 2017). A novel class of nanomedicines, high Z nanoparticle (NP) radiosensitisation agents, that possess the potential to further increase the efficacy of photon radiotherapy have recently entered the first phase of clinical trials

(c) The Author(s) 2018. This article is distributed under the terms of the Creative Commons Attribution 4.0 International License (http://creativecommons.org/licenses/by/4.0/), which permits unrestricted use, distribution, and reproduction in any medium provided you give appropriate credit to the original author(s) and the source, provide a link to the Creative Commons license, and indicate if changes were made. 
(National Institutes of Health (USA) 2016). These nanomedicines can be functionalised to seek out cancerous cells/tumours and when irradiated increase the local energy deposition and free-radical yield within a few tens to hundreds of nanometres surrounding each NP (Hainfeld et al. 2004, 2008; Jones et al. 2010; McMahon et al. 2011; Jain et al. 2012; Lechtman et al. 2013; Lin et al. 2014; Sicard-Roselli et al. 2014; Tran et al. 2016). However, the majority of in-vitro studies exploring the use of these nanomedicines for photon radiotherapy applications illustrate that the biological response of high Z NPdoped cells do not directly scale with these factors alone (McMahon et al. 2011; Lechtman et al. 2013; Chithrani et al. 2010; Liu et al. 2010; Jain et al. 2011; Sancey et al. 2014; Nicol 2016).

The complex nature of biological response of high Z NP-doped cells under irradiation from clinical photon radiotherapy sources presents a significant challenge when developing accurate treatment planning schemes. Initially, investigators attempted to apply a variety of Dose Enhancement Figures of Merit (DEFM) to evaluate the possible potential of high Z NP enhancement photon radiotherapy in the clinical setting (Cho 2005; Roeske et al. 2007; Ngwa et al. 2010). These DEFMs were based on the assumption that the expected biological outcome of cells/tumours could be described via the ratio of dose deposition with and without high Z NP doping under uniform photon irradiation, a concept now known to possess limited validity (McMahon et al. 2011; Lechtman et al. 2013; Chithrani et al. 2010; Liu et al. 2010; Jain et al. 2011; Sancey et al. 2014). For a high $\mathrm{Z}$ NP-enhanced photon radiotherapy treatment planning framework to be applicable to the clinical setting, it would need to account for the complex biological behaviour of NPdoped cells whilst conveying the improved outcome in terms comparable with conventional photon radiotherapy.

Recently Ferrero et al. (2017) and Strigari et al. (2017) presented a two article series on the treatment planning of radiotherapy for breast cancer using gold nanoparticles (AuNP). The first of these papers focused on the reformulation of the local effect model (LEM) to estimate AuNP-driven increase in radiosensitivity. Here a physics-driven additive approach was implemented based on the average number of ionisations per AuNP per Gy of dose, and the average number of AuNPs located inside or adjacent to the cell nucleus (Ferrero et al. 2017). In the second paper, this formalism was applied to the treatment planning of breast cancer radiotherapy with 2-nm-diameter AuNPs utilising Eclipse v.8.9 (Varian Medical Systems, Palo Alto, CA, USA) (Strigari et al. 2017). From these works, it was illustrated that an improved treatment efficacy outcome is possible based on the developed formalism and selected targeting proprieties of the AuNPs. However, as highlighted in Ferrero et al. this physics-driven approach neglects two major sets of physiological factors that underpin the biological response of NP-doped cells under irradiation: variation and localisation of AuNP uptake within/surrounding cells, and impact of AuNPs on toxicity and cellular cycle/repair processes (Ferrero et al. 2017).

This work proposes a biological data-driven framework, the Relative Biological Effective Dose (RBED), that aims to enable pre-clinical and clinical treatment planning of high Z NP-enhanced photon radiotherapy. It combines the Relative Biological Effectiveness $(\mathrm{RBE})$ phenomenological model for proton radiotherapy outlined in Wilkens and Oelfke (2004), with the experimentally benchmarked NP radiosensitisation interpolation 
framework of Brown and Currell (2017) to convey the observed increased biological effect in terms of conventional photon radiotherapy dose. The framework of Brown and Currell was developed to leverage available photon-NP biological radiosensitisation data to predict biological response with the specific purpose of accounting for the physiological effects associated with variation and localisation of AuNP uptake within/surrounding cells, and impact of NPs on toxicity and cellular cycle/repair processes. To illustrate the viability of the RBED framework as a pre-clinical/clinical treatment planning tool, an in-silico study of neoadjuvant (preoperative) high Z NP-enhanced breast cancer MV photon radiotherapy was undertaken utilising the Monte Carlo radiation transport modelling toolkit Geant4 (Agostinelli 2003; Allison 2006, 2016).

\section{Method and materials}

\section{Relative Biological Effective Dose (RBED) framework}

The RBED framework was developed to leverage available photon-NP biological radiosensitisation data to convey the increased biological response of high Z NP-enhanced photon radiotherapy on equal terms with conventional photon radiotherapy. In this work, two biological systems composed of the same cell line/tissue type are considered, one doped with a known concentration of a high $\mathrm{Z} \mathrm{NP}$ radiosensitising agent (System A) and one without (System B). The survival fraction of these two systems after irradiation, $\mathrm{SF}_{\mathrm{A}}$ for System A and $\mathrm{SF}_{\mathrm{B}}$ for System B, can be described:

$$
\begin{aligned}
& \mathrm{SF}_{\mathrm{A}}=\exp \left(-\alpha_{\mathrm{A}} D_{\mathrm{A}}-\beta_{\mathrm{A}} D_{\mathrm{A}}^{2}\right), \\
& \mathrm{SF}_{\mathrm{B}}=\exp \left(-\alpha_{\mathrm{B}} D_{\mathrm{B}}-\beta_{\mathrm{B}} D_{\mathrm{B}}^{2}\right),
\end{aligned}
$$

where $D_{\mathrm{A}}$ and $D_{\mathrm{B}}$ are the administered radiation doses, and $\alpha_{\mathrm{A}}, \alpha_{\mathrm{B}}, \beta_{\mathrm{A}}$ and $\beta_{\mathrm{B}}$ are the fitted Linear Quadratic (LQ) model (Douglas and Fowler 1976) parameters of each respective system. These systems can be said to have biological equivalence when the survival fraction of their irradiated cell populations are equal:

$$
\exp \left(-\alpha_{\mathrm{A}} D_{\mathrm{A}}-\beta_{\mathrm{A}} D_{\mathrm{A}}^{2}\right)=\exp \left(-\alpha_{\mathrm{B}} D_{\mathrm{B}}-\beta_{\mathrm{B}} D_{\mathrm{B}}^{2}\right)
$$

which, after further manipulation, yields

$$
\beta_{\mathrm{B}} D_{\mathrm{B}}^{2}+\alpha_{\mathrm{B}} D_{\mathrm{B}}-\left(\alpha_{\mathrm{A}} D_{\mathrm{A}}+\beta_{\mathrm{A}} D_{\mathrm{A}}^{2}\right)=0 .
$$

Therefore, the RBED of the system containing the high Z NPs can be given via the positive solution for $D_{\mathrm{B}}$ of this quadratic expression:

$$
D_{\mathrm{B}}=\frac{\sqrt{\alpha_{\mathrm{B}}^{2}+4 \beta_{\mathrm{B}}\left(\alpha_{\mathrm{A}} D_{\mathrm{A}}+\beta_{\mathrm{A}} D_{\mathrm{A}}^{2}\right)}-\alpha_{\mathrm{B}}}{2 \beta_{\mathrm{B}}}
$$

which is equivalent to Equation 3 in Wilkens and Oelfke (2004).

The key factor that dictates the accuracy of the phenomenological approach outlined above are the sourced values of each systems $\alpha$ and $\beta$. Whilst it is possible to obtain 
these values from a number different of sources, e.g. clonogenic cell assays, clinical trials, computational radiobiology, the majority of these high $\mathrm{Z} \mathrm{NP}$ radiosensitisation studies for given cell line/tissue type are typically limited to two measurements for each tested photon source: a control and a single doped concentration $\left(C_{\mathrm{M}}\right)$. To enable the application of these datasets to a clinically relevant scenario, i.e. varied high $\mathrm{Z} \mathrm{NP}$ concentrations $(C)$ in different tissue regions, interpolated values for $\alpha_{\mathrm{A}}$ and $\beta_{\mathrm{A}}$ can be determined using the interpolation framework of Brown and Currell (2017), i.e.,

$$
\alpha_{\mathrm{A}} D_{\mathrm{A}}+\beta_{\mathrm{A}} D_{\mathrm{A}}^{2}=\left(\alpha_{\mathrm{B}}+\frac{C}{C_{\mathrm{M}}} \Delta \alpha\right) D_{\mathrm{A}}+\left(\beta_{\mathrm{B}}+\frac{C}{C_{\mathrm{M}}} \Delta \beta\right) D_{\mathrm{A}}^{2},
$$

where $\Delta \alpha=\alpha_{\mathrm{B}}\left(C_{\mathrm{M}}\right)-\alpha_{\mathrm{A}}$ and $\Delta \beta=\beta_{\mathrm{B}}\left(C_{\mathrm{M}}\right)-\beta_{\mathrm{A}}$. Substitution of Eq. 6 into Eq. 5 gives the final form of the RBED framework:

$$
D_{\mathrm{B}}=\frac{\sqrt{\alpha_{\mathrm{B}}^{2}+4 \beta_{\mathrm{B}}\left(\left(\alpha_{\mathrm{B}}+\frac{C}{C_{\mathrm{M}}} \Delta \alpha\right) D_{\mathrm{A}}+\left(\beta_{\mathrm{B}}+\frac{C}{C_{\mathrm{M}}} \Delta \beta\right) D_{\mathrm{A}}^{2}\right)}-\alpha_{\mathrm{B}}}{2 \beta_{\mathrm{B}}} .
$$

\section{In-silico study of high Z NP-enhanced breast cancer MV photon radiotherapy}

An in-silico platform was developed to illustrate the potential of the RBED framework for the medical exemplar of neoadjuvant (preoperative) breast cancer MV photon radiotherapy (Calitchi et al. 2001; Riet et al. 2017) using the Monte Carlo radiation transport modelling toolkit Geant4 version 10.02.p02. The implemented simulation platform was designed to emulate the administration of a single 2 Gy fraction as part of a 50 Gy radiotherapy treatment plan across a two-compartment spherical tumour located in the left breast of an adult female. Two different treatment scenarios, conventional and high $\mathrm{Z}$ NP enhanced, were explored for a set of $1 \mathrm{~Gy}$ on-axis tumour-shaped projections with a 6 MV Varian Linac photon source. For the high Z NP-enhanced treatment scenario, it was assumed that a sufficient quantity of the popular commercial proprietary thiolcapped high Z NP radiosensitisation agent Aurovist (Hainfeld et al. 2004; Jain et al. 2011, 2012; Rahman et al. 2009; Bobyk et al. 2013; Al Zaki et al. 2014; Her et al. 2017), 1.9-nmdiameter gold nanoparticles (AuNPs), was administered to the patient to yield a maximum concentration within the two-compartment tumour of $500 \mu \mathrm{g} / \mathrm{ml}$.

Figure 1 presents a set of cross-sectional views of the simulated adult female phantom. The simulated adult female phantom was composed of a soft tissue elliptical cylinder containing the $\mathrm{C} 1$ to $\mathrm{C} 7$ vertebra of the spine, sternum, the seven attached ribs, both lungs and solid skewed ellipsoid representing the heart. Two fused cylinder-ellipsoids representing the breasts were contoured onto the surface of the elliptical cylinder centred in front of the third rib. Each breast is rotated $20^{\circ}$ way from the sternum to mimic their resting position for a patient lying on their back. Within the left breast, a two-compartment tumour of diameters $20 \mathrm{~mm}$ and $16 \mathrm{~mm}$ is located $20 \mathrm{~mm}$ in front of the breast centre away from the torso. In the case of the high $\mathrm{Z}$ NP-enhanced simulated irradiation, a uniform AuNP concentration of $250 \mu \mathrm{g} / \mathrm{ml}$ and $500 \mu \mathrm{g} / \mathrm{ml}$ was implemented for the inner and tumour wall regions, respectively, to approximate the uptake effects observed within solid tumours (Brown and Giaccia 1998; Minchinton and Tannock 2006; Hainfeld et al. 2011), whereas the remaining soft tissue and organs was set to $25 \mu \mathrm{g} / \mathrm{ml}$ to emulate the small animal uptake 

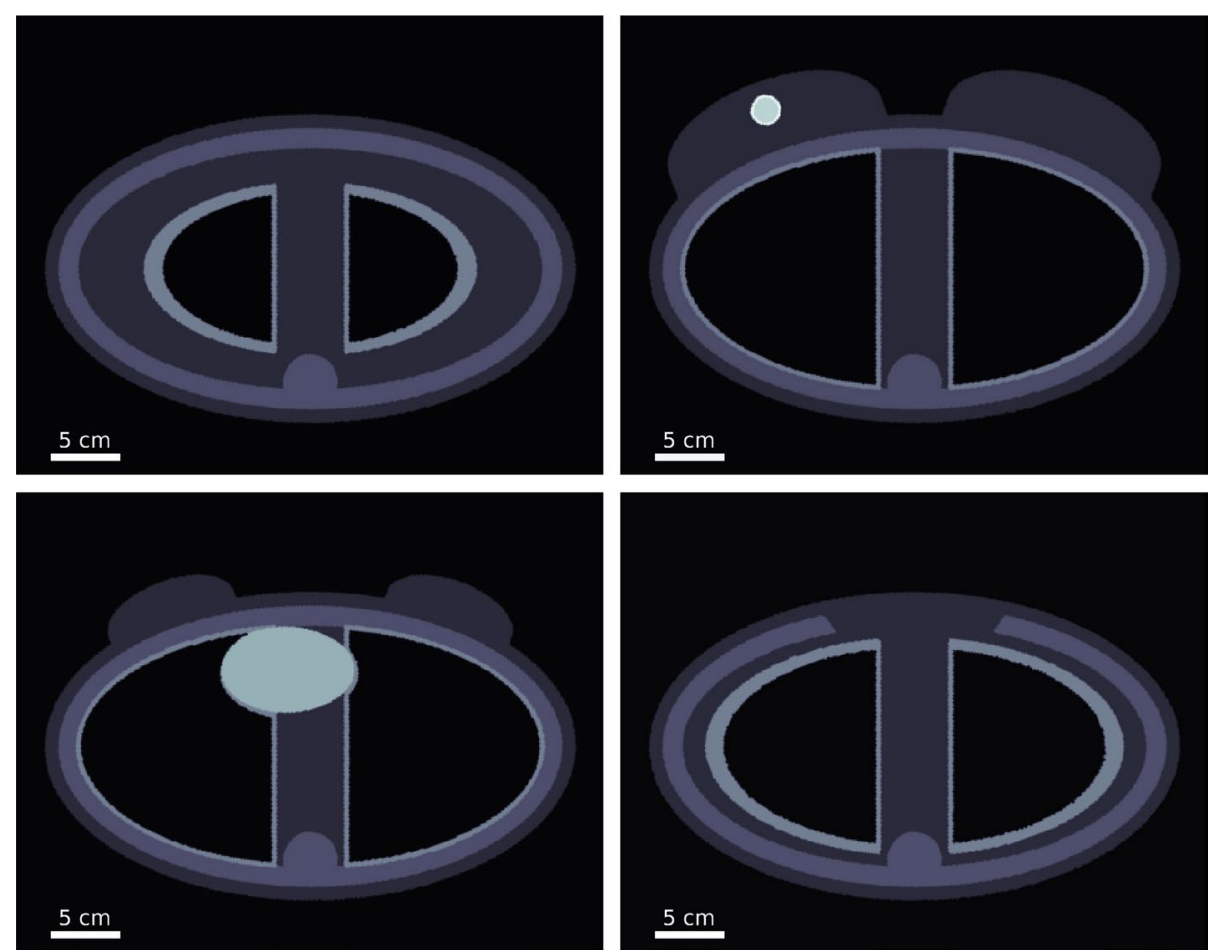

Fig. 1 Cross-sectional view of the simulated adult female phantom at the centre of the first (top left), third (top right), fourth (bottom left) and sixth ribs (bottom right). In the top left and bottom right images, top and bottom cross sections of the lung volume can be seen, respectively. The top right cross section displays the central axis of the breasts and the two-compartment tumour in addition to the lung walls, whereas the bottom left cross section displays the central axis of the solid skewed ellipsoid representing the heart in addition to the lung walls. A voxelised version of the phantom can be obtained at the Delft University of Technology Research Data repository (http://researchdata.4tu.nl)

distributions for the Aurovist radiosensitisation agents observed in Hainfeld et al. $(2006,2011)$. A summary of the material types of the different phantom components and their relative AuNP concentrations is outlined in Table 1.

To achieve a minimum 2 Gy dose across the tumour and limit dose to non-tumour regions within the simulated phantom, the irradiation geometry, seen in Fig. 2 (left), was composed of a 30-mm-diameter circular non-diverging photon beam rotated $20^{\circ}$ off-axis focused at the two-compartment tumour centre. Both treatment scenarios implemented two equal "exposure time" sub-fraction irradiations delivered in opposite directions along the dashed axis seen in Fig. 2 (left). Each sub-fraction irradiation simulated a total of $1.55 \times 10^{12}$ primary photons of energy sampled from the $6 \mathrm{MV}$ Varian Linac photon energy spectra presented in Fig. 2 (right). These simulations were undertaken at the macroscopic scale with $1 \mathrm{~mm}^{3}$ Dose Volume Histogram (DVH) voxelisation using the Geant4 Option4 EM physics configuration. Atomic deexcitation, a maximum particle step length of $250 \mu \mathrm{m}$ and a low-energy particle cutoff of $1 \mathrm{keV}$, was implemented to simulate the transport of primary photons and their generated secondary particles throughout the phantom.

The radiosensitisation response parameters, i.e. $\alpha$ and $\beta$ values, for the AuNP-doped tissue types of the phantom were sourced from Jain et al. (2011). This study explored 
Table 1 Material type and ratio of AuNP uptake of each phantom component seen in Fig. 1

\begin{tabular}{|c|c|c|}
\hline Phantom component & Material & $\begin{array}{l}\text { AuNP } \\
\text { uptake } \\
(\mu \mathrm{g} / \\
\mathrm{ml})\end{array}$ \\
\hline Ribs & $\begin{array}{l}\text { ICRU-49 Compact Bone International Commission on Radiation Units and } \\
\text { Measurements (1994) }\end{array}$ & 0 \\
\hline Spine & $\begin{array}{l}\text { ICRU-49 Compact Bone International Commission on Radiation Units and } \\
\text { Measurements (1994) }\end{array}$ & 0 \\
\hline Sternum & $\begin{array}{l}\text { ICRU-49 Compact Bone International Commission on Radiation Units and } \\
\text { Measurements (1994) }\end{array}$ & 0 \\
\hline Heart & $\begin{array}{l}\text { ICRU-44 Muscle International Commission on Radiation Units and Measure- } \\
\text { ments (1989) }\end{array}$ & 25 \\
\hline Lung wall & $\begin{array}{l}\text { ICRU-44 Lung Tissue International Commission on Radiation Units and } \\
\text { Measurements (1989) }\end{array}$ & 25 \\
\hline Torso bulk & $\begin{array}{l}\text { ICRU-44 Soft Tissue International Commission on Radiation Units and Meas- } \\
\text { urements (1989) }\end{array}$ & 25 \\
\hline Breast & $\begin{array}{l}\text { ICRU-44 Breast Tissue International Commission on Radiation Units and } \\
\text { Measurements (1989) }\end{array}$ & 25 \\
\hline Tumour inner & $\begin{array}{l}\text { ICRU-44 Breast Tissue International Commission on Radiation Units and } \\
\text { Measurements (1989) }\end{array}$ & 250 \\
\hline Tumour wall & $\begin{array}{l}\text { ICRU-44 Breast Tissue International Commission on Radiation Units and } \\
\text { Measurements (1989) }\end{array}$ & 500 \\
\hline
\end{tabular}

A voxelised version of the phantom can be obtained at the Delft University of Technology Research Data repository (http:// researchdata.4tu.nl)
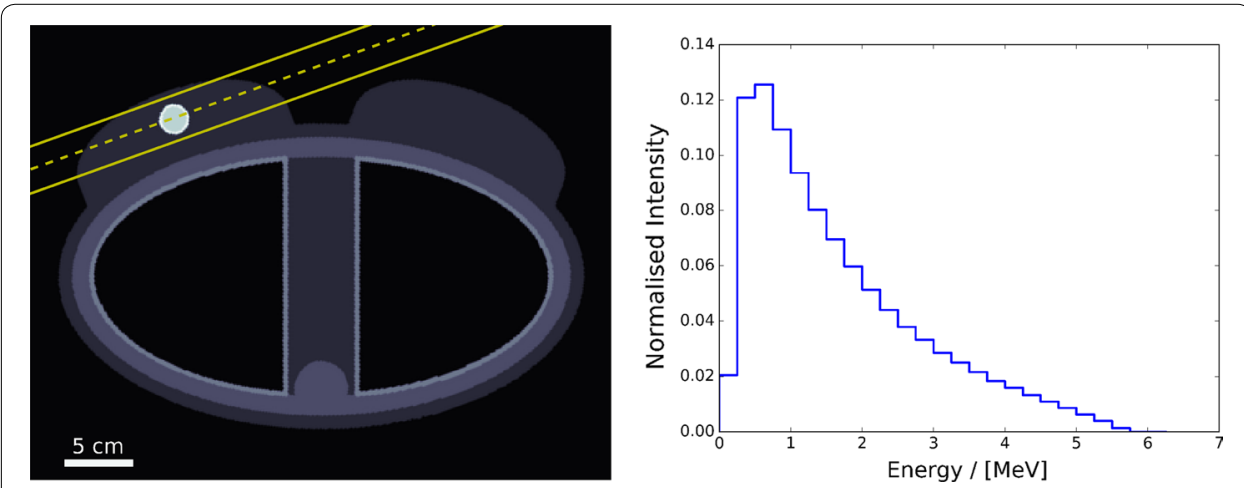

Fig. 2 Cross-section of the simulated adult female phantom irradiation geometry (left) and primary photon energy spectra of the simulated 6 MV Varian Linac source taken from Sheikh-Bagheri and Rogers (2002) (right). The solid and dashed yellow lines represent the outer edges and central axis, respectively, of the 30 -mm-diameter circular non-diverging photon beam rotated $20^{\circ}$ off-axis focused at the two-compartment tumour centre located in the left breast

the radiosensitisation of three different human cell lines, DU145 prostate cancer cells, MDA-MB-231 breast cancer cells and L132 lung epithelial cells, for the selected AuNP agent and discovered that only the AuNP-doped MDA-MB-231 breast cancer cells saw increased sensitisation at megavoltage energies (6 MV and $15 \mathrm{MV}$ ). Based on these findings, and the fact that the expected AuNP uptake in health tissue is significantly lower than the tumour due to enhanced permeability and retention effects (Maeda 2012), it was assumed that only breast cells undergo an increased radiosensitisation with the selected AuNP agent at megavoltage photon energies. Therefore 
the heart, lung and soft tissue regions within the phantom were modelled as having a zero increase in radiosensitisation, whereas the breast tissue that makes up both breast and the tumour regions were modelled using the 6MV Varian Linac-irradiated MDA-MB-231 breast cancer cell parameters contained in Table 1 of Jain et al. (2011). To reflect the inherent uncertainty in-vitro clonogenic assays experiments, and the fundamental difference between cell culture and tissue response under irradiation, three different scenarios of radiobiological response was explored: minimum, mean and maximum relative AuNP agent radiosensitisation (see Table 2 for their $\alpha$ and $\beta$ parameter values).

\section{Results}

Dose deposition within the conventional and AuNP-doped phantom

Figure 3 presents dose maps of the adult female phantom, both conventional and AuNP doped, and their parallel and perpendicular dose profiles with respect to the simulated central axis of the photon beam. Inspection of these dose maps and profiles illustrate that (1) in each treatment scenario the majority of the dose deposited within the phantom was limited to the target region defined via the edges of the shaped photon field outline in Fig. 2, and (2) the dose deposition in both the conventional and AuNP doped are effectively identical resulting in the overlap of their respective parallel and perpendicular profiles. The accompanying parallel dose profiles further illustrate that, as intended, the dose delivered throughout the left breast along the central axis of irradiation exceeded 2 Gy in both treatment scenarios, whereas the perpendicular dose profiles show the presence of a dose gradient across the central target region of the photon beam, $\pm 10 \mathrm{~mm}$, from left to right that approaches 2 Gy at the tumour's right edge. This gradient is the result of two primary physical factors: different extents of spectral hardening and intensity variation of the beam incident on the tumour surface [due to varied path lengths of beam through the left breast (Metcalfe et al. 1990)], and beam broadening due to photon scattering [illustrated via the Gaussian like roll-off after $\pm 15 \mathrm{~mm}$ (Ahnesjö and Aspradakis 1999)]. However, even with these factors the minimum dose administered to the whole tumour in both simulated treatment scenarios reached the desired target of 2 Gy (see Fig. 4).

Quantification of the observed minimal difference in the dose deposited between the two treatment scenarios can be visualised via each simulated phantom's DVH, and minimum, mean and maximum breast tissue and whole tumour/tumour region

Table 2 Phantom breast tissue $\alpha$ and $\beta$ values for the minimum, mean and maximum radiosensitisation response scenarios for high Z NP-enhanced photon radiotherapy with $1.9 \mathrm{~nm}$ Aurovist AuNPs under $6 \mathrm{MV}$ Linac irradiation

\begin{tabular}{|c|c|c|c|c|}
\hline Scenario & $\begin{array}{l}\alpha \\
(0 \mu \mathrm{g} / \mathrm{ml})\end{array}$ & $\beta$ & $\begin{array}{l}\alpha \\
(500 \mu \mathrm{g} / \mathrm{ml})\end{array}$ & $\beta$ \\
\hline Minimum & 0.024 & 0.086 & 0.064 & 0.087 \\
\hline Mean & 0.002 & 0.079 & 0.104 & 0.098 \\
\hline Maximum & 0.000 & 0.072 & 0.144 & 0.109 \\
\hline
\end{tabular}

Data taken from Jain et al. (2011) MDA-MB-231 breast cancer cell response. Minimum case $\alpha$ and $\beta$ determine by minimising AuNP-doped and maximising conventional cell response within experimental uncertainty. Maximum case $\alpha$ and $\beta$ determine by maximising AuNP-doped and minimising conventional cell response within experimental uncertainty 

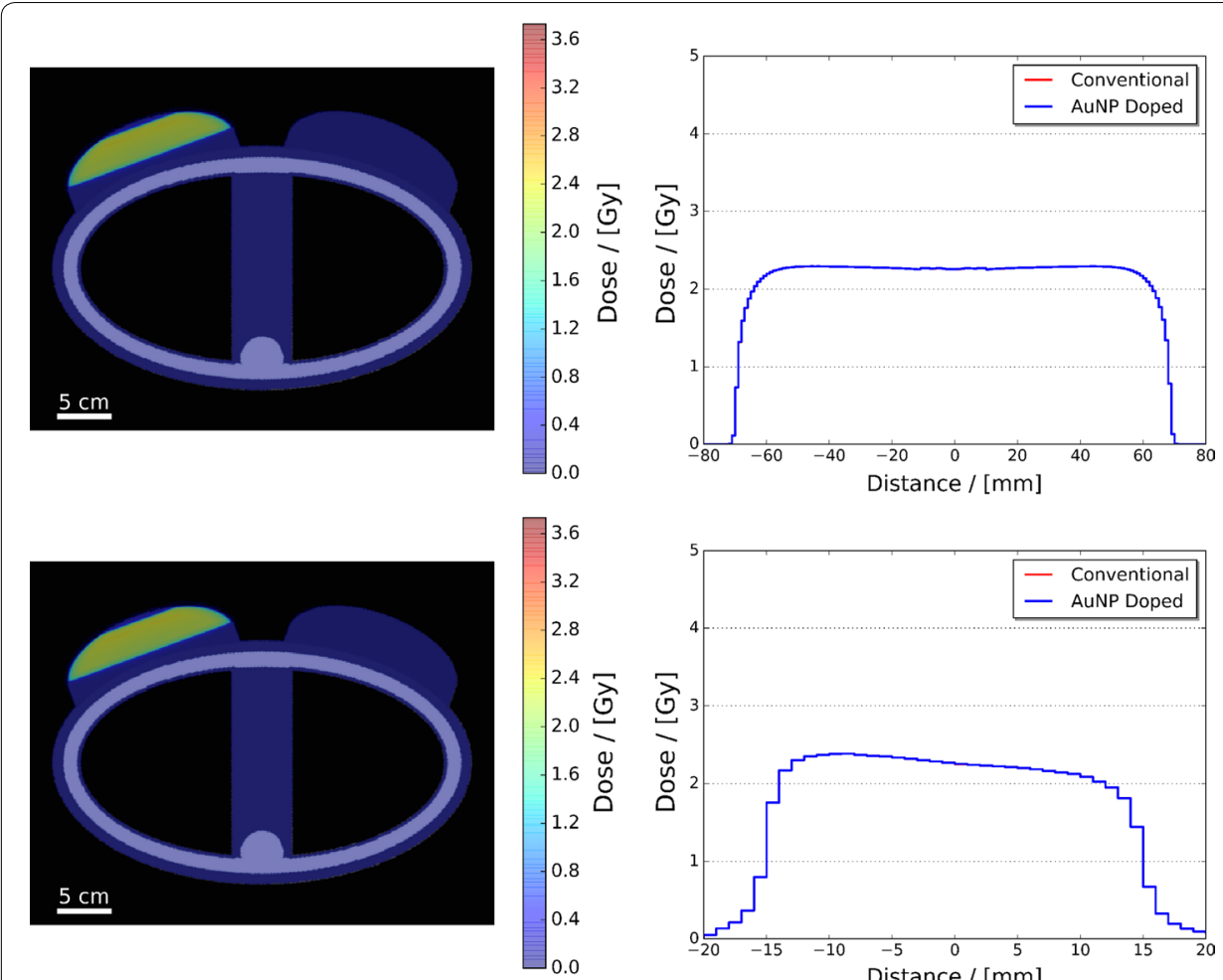

Fig. 3 Dose maps of the adult female phantom, conventional (top left) and AuNP doped (bottom left), and accompanying parallel (top right) and perpendicular (bottom right) dose profiles with respect to the simulated central axis of the photon beam
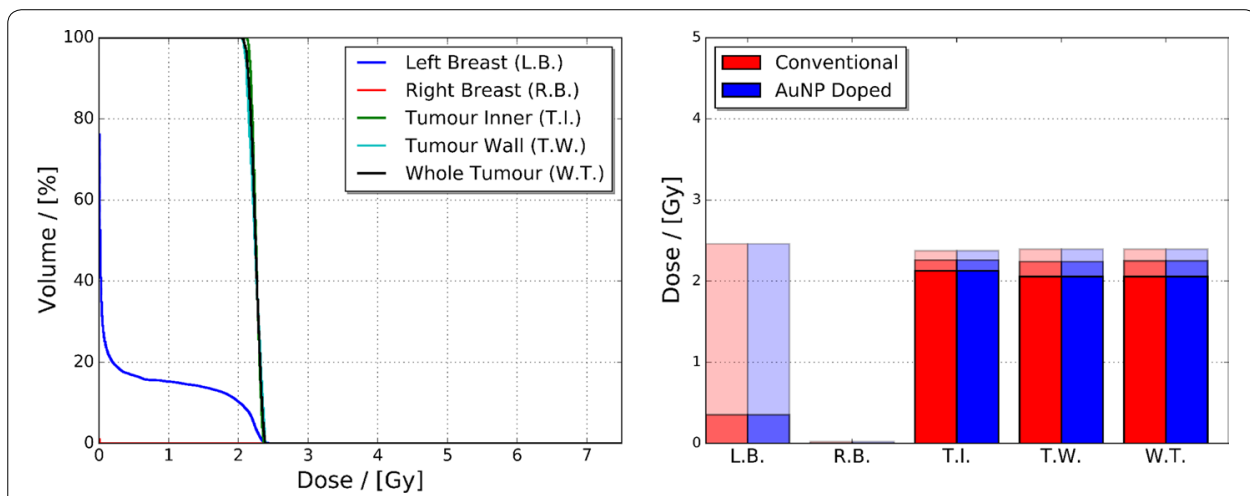

Fig. 4 Dose Volume Histograms (DVHs) (left), and minimum, mean and maximum dose (right), illustrated via increasing transparency, of the simulated conventional and AuNP-doped adult female phantom breast tissue and whole tumour/tumour regions. The conventional (solid) and AuNP-doped (dashed) DVH profiles for the breast tissue and tumour regions differ by less than $1 \%$ causing an overlap

delivered doses seen in Fig. 4. Comparison of each phantom regions DVHs and mean dose illustrates that there is less than $1 \%$ difference between the two treatment scenarios (which is on the same order of magnitude as the statistical accuracy of the simulation). Furthermore, both sets of DVHs illustrate that near-zero dose is deposited outside of the left breast and target tumour region. 


\section{RBED within the conventional and AuNP-doped phantom}

Figure 5 presents the RBED maps of the irradiated adult female phantom for the conventional, minimum, mean and maximum relative AuNP agent radiosensitisation scenarios. With increasing relative radiosensitisation, the inner and tumour wall regions display an increased biological effect with respect to conventional radiotherapy (as indicated via increased RBED). The accompanying parallel and perpendicular RBED profiles of these maps further convey the extent of these increases within the inner and tumour wall regions of up to 3.08 and 3.67 Gy for the maximum radiosensitisation scenario, respectively. However, these RBED profiles also illustrate an increased biological effect in the left breast that scales proportionally with relative AuNP radiosensitisation. This observable increase within the left breast, which was less than $10 \%$ for all three relative radiosensitisation scenarios, highlights the need for correct selection of the high Z NP agent

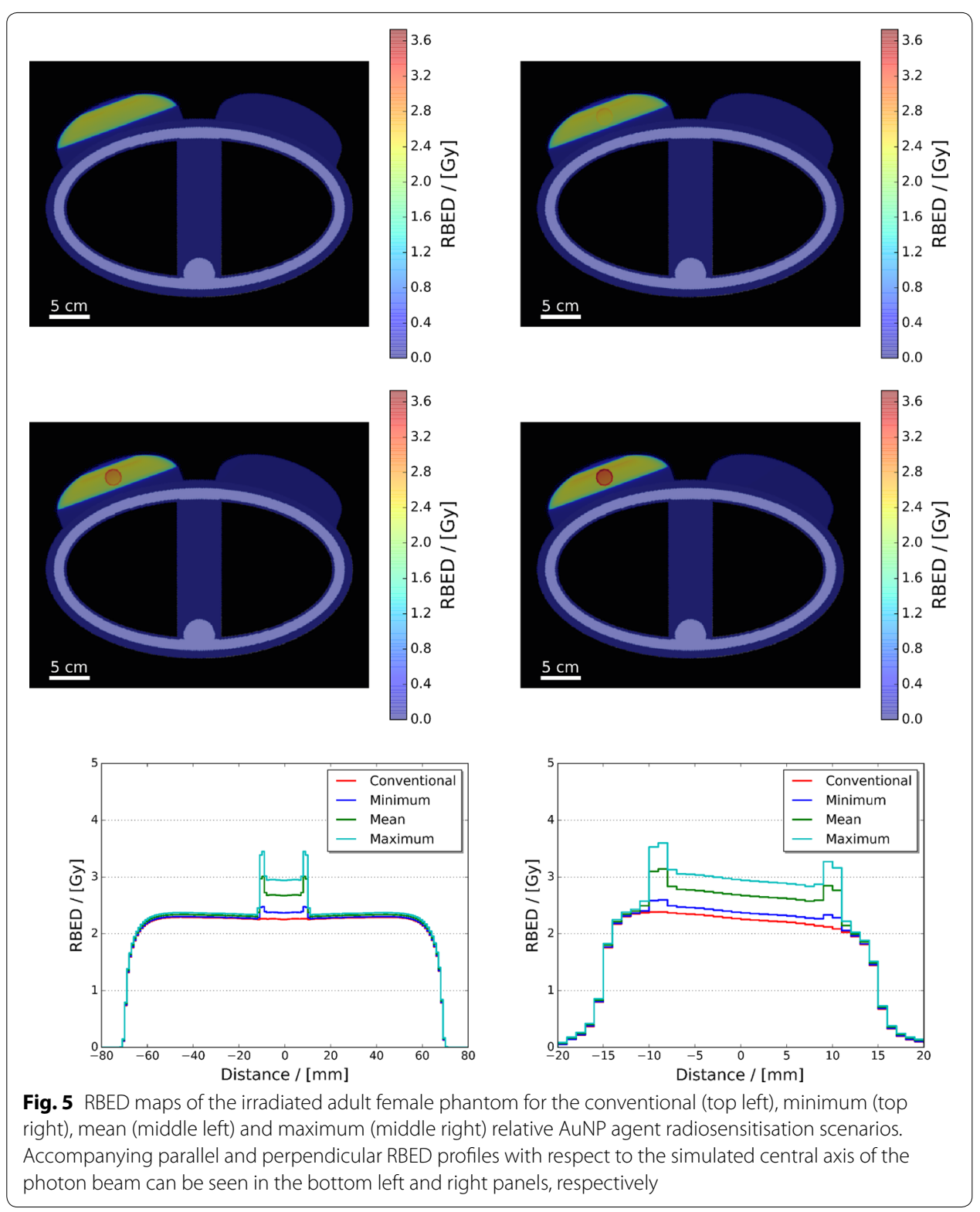


to ensure maximised uptake in the target irradiation volume whilst minimising uptake in the surrounding non-target tissue. Accounting for this effect is expected to be one of the most important considerations in high Z NP-enhanced photon radiotherapy treatment planning to ensure maximum tumour control probability whilst minimising normal tissue complications.

The RBED Volume Histograms (RVHs), and minimum, mean and maximum breast tissue and whole tumour/tumour region RBEDs for the conventional, minimum, mean and maximum relative AuNP agent radiosensitisation scenarios can be seen in Fig. 6 . The observed trends in the RBED maps and profiles of Fig. 5 correlate with the increased RBED per unit volume of the left breast and tumour regions displayed in the RVHs. The extent of increase in mean RBED from the conventional to maximum relative AuNP agent radiosensitisation for the left and right breasts spans from 0.354 to 0.390 Gy and 0.002 to 0.012 Gy, respectively, whereas for the tumour inner, tumour wall and whole tumour the mean RBED increases from 2.258 to $2.945 \mathrm{~Gy}, 2.241$ to $3.478 \mathrm{~Gy}$, and 2.250 to $3.190 \mathrm{~Gy}$.

\section{Discussion}

A novel framework for pre-clinical and clinical treatment planning of high Z NPenhanced photon radiotherapy was developed and its applicability tested in silico for the medical exemplar of neoadjuvant (preoperative) cancer MV photon radiotherapy. The

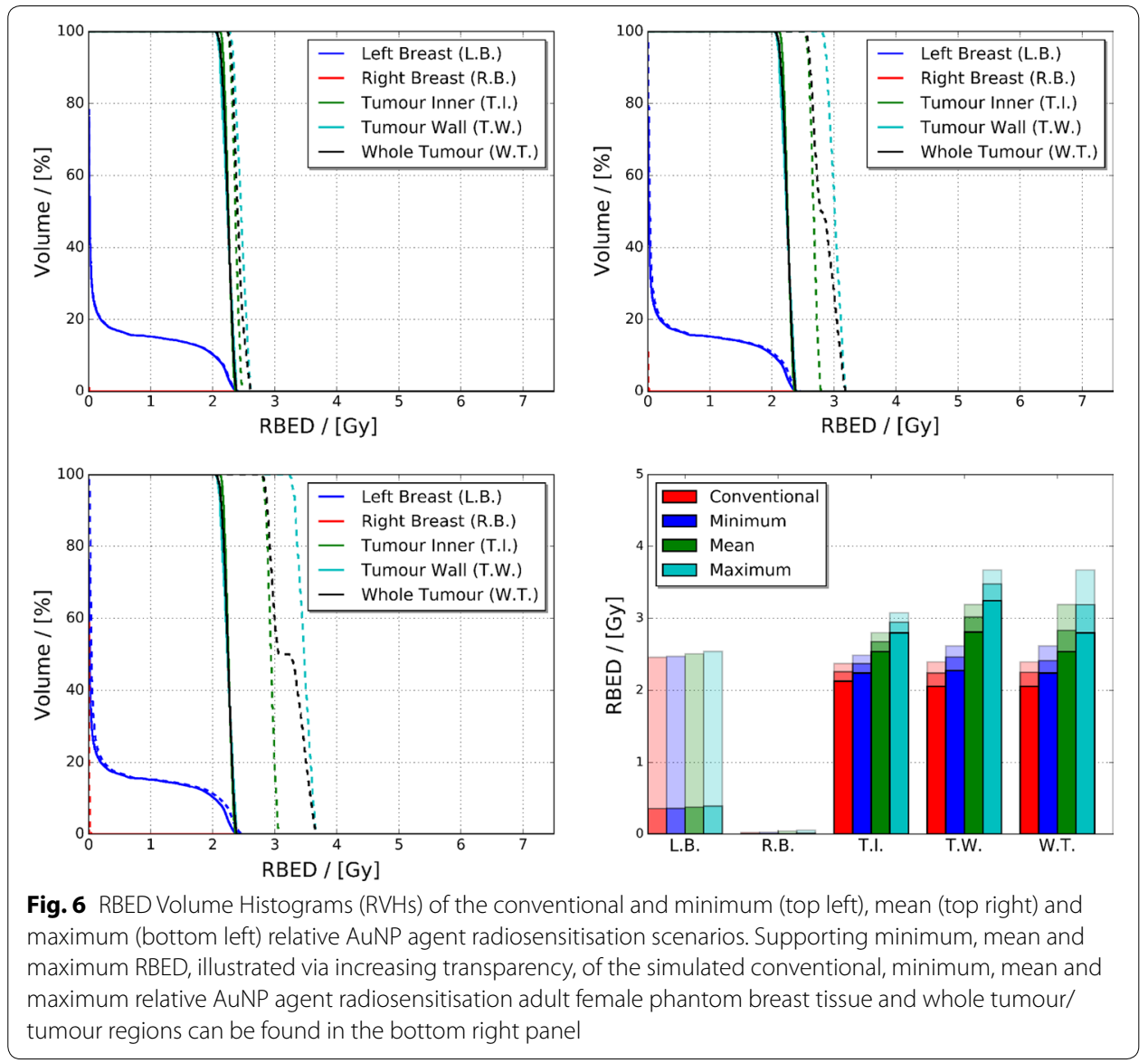


simulations undertaken with the developed in-silico platform illustrated that for a semirealistic uptake distribution of the selected AuNP radiosensitisation agent there was less than a $1 \%$ difference in the dose deposition throughout the standard and AuNP-doped adult female phantom. Application of the RBED framework for the three different relative AuNP agent radiosensitisation scenarios highlight that, even for this near-zero dose deposition difference, in every case an increase biological response was present in the left breast, right breast and tumour with respect to conventional radiotherapy. In fact, to achieve the same level of biological response within the tumour as the minimum, mean and maximum radiosensitisation scenarios, the conventional radiotherapy treatment approach would require a 9\%,33\% and 53\% increase in radiation exposure, respectively. Overall these results further illustrate the need of a framework such as RBED which is capable of accounting for the complex biological behaviour of NP-doped cells whilst conveying the improved outcome in terms comparable with conventional photon radiotherapy.

Whilst the findings of the present work point to the viability of the selected AuNP radiosensitisation agent for $\mathrm{MV}$ photon radiotherapy treatment of neoadjuvant breast cancer, due to a number of approximations it is difficult to draw a definite conclusion. Within the simulated adult female phantom, the distribution of the high Z NP radiosensitisation agent uptake was simplified to a generic geometry due to the lack of supporting patient data. In reality, it is expected that the distribution of the high Z NP agent uptake within the patent will differ throughout various tissue regions and the target tumour due to its complex vascular structure (Brown and Giaccia 1998; Minchinton and Tannock 2006). Moreover, the limited nature of available photon-NP biological radiosensitisation data forced a number of assumptions to be employed for the soft tissue response of the selected high Z NP agent under MV photon irradiation. If further supporting photonNP biological radiosensitisation data and anatomical uptake distributions within realistic patient geometries could be obtained, it would be possible via the RBED framework to make an informed conclusion. However, the aim of this work was to introduce the RBED framework to the scientific community and illustrate that, with appropriate supporting data, questions such as these could be explored via both in-silico and experimental trials.

\section{Conclusion}

A novel framework for the pre-clinical and clinical treatment planning of high Z NPenhanced photon radiotherapy was developed and its applicability tested in silico for the medical exemplar of neoadjuvant (preoperative) breast cancer MV photon radiotherapy. Whilst a definite conclusion about the viability of the selected high Z NP radiosensitisation agents for the selected photon radiotherapy medical exemplar could not be obtained, it was illustrated with the RBED framework that the extent of possible biological response was great than expected via the dose deposition alone. Further exploration of the role of high Z NP radiosensitisation agents in photon radiotherapy is warranted and it is anticipated that this framework will assist the scientific community in future high Z NP-enhanced in-silico, pre-clinical and clinical trials. 


\section{Abbreviations}

AuNP:: gold nanoparticle; DEFM:: dose enhancement figures of merit; NP:: nanoparticle; LEM::: local effect model; RBE:: Relative Biological Effectiveness; RBED:: Relative Biological Effective Dose.

\section{Authors' contributions}

JMCB developed the theory, developed the simulation platform, designed and undertook the analysis, and wrote/edited the manuscript. GGH contributed to the development of the simulation platform, clinical relevance of the simulations, and edited the manuscript. NL contributed to the analysis, biological relevance of the simulations, and edited the manuscript. BVB contributed to the clinical relevance of the simulations, and edited the manuscript. JRN and JAC contributed to the biological relevance of the simulations, and edited the manuscript. FJC commissioned the project, mentored $J M C B$ and edited the manuscript. All authors read and approved the final manuscript.

\section{Author details}

${ }^{1}$ School of Mathematics and Physics, Queen's University Belfast, Belfast, Northern Ireland, UK. ${ }^{2}$ Department of Radiation Science and Technology, Delft University of Technology, Delft, The Netherlands. ${ }^{3}$ Centre for Medical Radiation Physics, University of Wollongong, Wollongong, Australia. ${ }^{4}$ School of Medicine, Dentistry and Biomedical Sciences, Queen's University Belfast, Belfast, Northern Ireland, UK. ${ }^{5}$ Université Clermont Auvergne, CNRS/IN2P3, LPC, Clermont-Ferrand, France. ${ }^{6}$ School of Pharmacy, Queen's University Belfast, Belfast, Northern Ireland, UK.

\section{Competing interests}

The authors declare that they have no competing interests.

\section{Data and materials}

Supporting data sets are accessible via the Delft University of Technology Research Data repository (http://researchda ta.4tu.nl).

\section{Funding}

This work was supported by EPSRC grant EP/K039342/1; and the Multi-modal Australian ScienceS Imaging and Visualization Environment (MASSIVE) (http://www.massive.org.au).

\section{Publisher's Note}

Springer Nature remains neutral with regard to jurisdictional claims in published maps and institutional affiliations.

Received: 2 July 2018 Accepted: 30 October 2018

Published online: 09 November 2018

\section{References}

Agostinelli S. GEANT4: simulation toolkit. Nuclear Instrum Methods Phys Res Sect A. 2003;506(3):250-303.

Ahnesjö A, Aspradakis MM. Dose calculations for external photon beams in radiotherapy. Phys Med Biol. 1999;44(11):R99-155.

Al Zaki A, Joh D, Cheng Z, De Barros ALB, Kao G, Dorsey J, Tsourkas A. Gold-loaded polymeric micelles for computed tomography-guided radiation therapy treatment and radiosensitization. Acs Nano. 2014;8(1):104-12.

Allison J. Geant4 developments and applications. IEEE Trans Nuclear Sci. 2006;53(1):270-8.

Allison J. Recent developments in Geant4. Nuclear Instrum Methods Phys Res Sect A. 2016:835:186-225.

Aupérin A, Le Péchoux C, Rolland E, Curran WJ, Furuse K, Fournel P, Belderbos J, Clamon G, Ulutin HC, Paulus R, Yamanaka T. Meta-analysis of concomitant versus sequential radiochemotherapy in locally advanced non-small-cell lung cancer. J Clin Oncol. 2010;28(13):2181-90.

Bobyk L, Edouard M, Deman P, Vautrin M, Pernet-Gallay K, Delaroche J, Adam JF, Estève F, Ravanat JL, Elleaume H. Photoactivation of gold nanoparticles for glioma treatment, nanomedicine: nanotechnology. Biol Med. 2013;9(7):1089-97.

Brown JMC, Currell FJ. A local effect model-based interpolation framework for experimental nanoparticle radiosensitisation data. Cancer Nanotechnol. 2017;8:1.

Brown JM, Giaccia AJ. The unique physiology of solid tumors: opportunities (and problems) for cancer therapy. Cancer Res. 1998;58(7):1408-16

Calitchi E, Kirova YM, Otmezguine Y, Feuilhade F, Piedbois Y, Le Bourgeois JP. Long-term results of neoadjuvant radiation therapy for breast cancer. Int J Cancer. 2001;96(4):253-9.

Chen G, Roy I, Yang C, Prasad PN. Nanochemistry and nanomedicine for nanoparticle-based diagnostics and therapy. Chem Rev. 2016;116(5):2826-85.

Chithrani DB, Jelveh S, Jalali F, van Prooijen M, Allen C, Bristow RB, Hill RP, Jaffray DA. Gold nanoparticles as radiation sensitizers in cancer therapy. Radiat Res. 2010;173(6):719-28.

Cho SH. Estimation of tumour dose enhancement due to gold nanoparticles during typical radiation treatments: a preliminary Monte Carlo study. Phys Med Biol. 2005;50(15):N163.

Delaney G, Jacob S, Featherstone C, Barton M. The role of radiotherapy in cancer treatment: estimating optimal utilization from a review of evidence-based clinical guidelines. Cancer. 2005;104(6):1129-37.

Douglas BG, Fowler JF. The effect of multiple small doses of X-rays on skin reactions in the mouse and a basic interpretation. Radiat Res. 1976;66(2):401-26.

Ferrero V, Visonà G, Dalmasso F, Gobbato A, Cerello P, Strigari L, Visentin S, Attili A. Targeted dose enhancement in radiotherapy for breast cancer using gold nanoparticles, part 1: a radiobiological model study. Med Phys. 2017:44(5):1983-92.

Hainfeld JF, Slatkin DN, Smilowitz HM. The use of gold nanoparticles to enhance radiotherapy in mice. Phys Med Biol, 2004;49(18):N309-15. 
Hainfeld JF, Slatkin DN, Focella TM, Smilowitz HM. Gold nanoparticles: a new X-ray contrast agent. Br J Radiol. 2006;79(939):248-53.

Hainfeld JF, Dilmanian FA, Slatkin DN, Smilowitz HM. Radiotherapy enhancement with gold nanoparticles. J Pharm Pharmacol. 2008;60(8):977-85.

Hainfeld JF, O'Connor MJ, Dilmanian FA, Slatkin DN, Adams DJ, Smilowit HM. Micro-CT enables microlocalisation and quantification of Her2-targeted gold nanoparticles within tumour regions. Br J Radiol. 2011;84:526-33.

Her S, Jaffray DA, Allen C. Gold nanoparticles for applications in cancer radiotherapy: mechanisms and recent advancements. Adv Drug Deliv Rev. 2017;109:84-101.

International Commission on Radiation Units and Measurements. ICRU report 44: tissue substitutes in radiation dosimetry and measurement. MD, USA: Bethesda; 1989.

International Commission on Radiation Units and Measurements. ICRU report 49: stopping power and ranges for Protons and Alpha particles. MD, USA: Bethesda; 1994.

Jain S, Coulter JA, Hounsell AR, Butterworth KT, MCMahon SJ, Hyland WB, Muir ME, Dickson GR, Prise KM, Currell EJ, O'Sullivan JM, Hirst DG. Cell-specific radiosensitization by gold nanoparticles at megavoltage radiation energies. Int J Radiat Oncol Biol Phys. 2011;79(2):531-9.

Jain S, Hirst DG, O'Sullivan JM. Gold nanoparticles as novel agents for cancer therapy. Br J Radiol. 2012;85:101-13.

Joiner M, van der Kogel A. Basic clinical radiobiological. 4th ed. Boca Raton: CRC Press, Taylor \& Francis Group; 2009

Jones BL, Krishnan S, Cho SH. Estimation of microscopic dose enhancement factor around gold nanoparticles by Monte Carlo calculations. Med Phys. 2010;37(7):3809-16.

Lechtman E, Mashouf S, Chattopadhyay N, Keller BM, Lai P, Cai Z, Reilly RM, Pignol JP. A Monte Carlo-based model of gold nanoparticle radiosensitization accounting for increased radiobiological effectiveness. Phys Med Biol. 2013;58(10):3075-87.

Lin Y, McMahon SJ, Scarpelli M, Paganetti H, Schuemann J. Comparing gold nano-particle enhanced radiotherapy with protons, megavoltage photons and kilovoltage photons: a Monte Carlo simulation. Phys Med Biol. 2014;59(24):7675-89.

Liu CJ, Wang CH, Chen ST, Chen HH, Leng WH, Chien CC, Wang CL, Kempson IM, Hwu Y, Lai TC, Hsiao M. Enhancement of cell radiation sensitivity by pegylated gold nanoparticles. Phys Med Biol. 2010;55(4):931-45.

Maeda H. Macromolecular therapeutics in cancer treatment: the EPR effect and beyond. J Control Release. 2012;164(2):138-44.

Marples B, Dhar S. Radiobiology and the renewed potential for nanoparticles. Int J Radiat Oncol Biol Phys. 2017:98(3):489-91.

Mayles P, Nahum A, Rosenwald J-C. Handbook of radiotherapy physics: theory and practice. New York: CRC Press, Taylor \& Francis Group; 2007.

McMahon SJ, Hyland WB, Muir MF, Coulter JA, Jain S, Butterworth KT, Schettino G, Dickson GR, Hounsell AR, O'Sullivan JM, Prise KM, Hirst DG, Currell FJ. Biological consequences of nanoscale energy deposition near irradiated heavy atom nanoparticles. Sci Rep. 2011;1:18.

Metcalfe PE, Hoban PW, Murray DC, Round WH. Beam hardening of 10 MV radiotherapy X-rays: analysis using a convolution/superposition method. Phys Med Biol. 1990;35(11):1533-49.

Minchinton Al, Tannock IF. Drug penetration in solid tumours. Nat Rev Cancer. 2006;6(8):583-92.

National Institutes of Health (USA). ClinicalTrials.gov. https://clinicaltrials.gov/. Accessed Dec 2016.

Ngwa W, Makrigiorgos GM, Berbeco RI. Applying gold nanoparticles as tumor-vascular disrupting agents during brachytherapy: estimation of endothelial dose enhancement. Phys Med Biol. 2010;55(21):6533-48.

Nicol J. Radiation dose enhancement: the development and application of radiosensitising gold nanoparticles, Doctoral Dissertation, Queen's University Belfast. United Kingdom: Northern Ireland; 2016.

Rahman WN, Bishara N, Ackerly T, He CF, Jackson P, Wong C, Davidson R, Geso M. Enhancement of radiation effects by gold nanoparticles for superficial radiation therapy, nanomedicine: nanotechnology. Biol Med. 2009;5(2):136-42.

Riet FG, Fayard F, Arriagada R, Santos MA, Bourgier C, Ferchiou M, Heymann S, Delaloge S, Mazouni C, Dunant A, Rivera S. Preoperative radiotherapy in breast cancer patients: 32 years of follow-up. Eur J Cancer. 2017;76:45-51.

Roeske JC, Nuñez L, Hoggarth M, Labay E, Weichselbaum RR. Characterization of the theoretical radiation dose enhancement from nanoparticles. Technol Cancer Res Treat. 2007;6(5):395-401.

Sancey L, Lux E, Kotb S, Roux S, Dufort S, Bianchi A, Cremillieux Y, Fries P, Coll JL, Rodriguez-Lafrasse C, Janier M. The use of theranostic gadolinium-based nanoprobes to improve radiotherapy efficacy. Br J Radiol. 2014;87(1041):20140134.

Schuemann J, Berbeco R, Chithrani DB, Cho SH, Kumar R, McMahon SJ, Sridhar S, Krishnan S. Roadmap to clinical use of gold nanoparticles for radiation sensitization. Int J Radiat Oncol Biol Phys. 2016;94(1):189-205.

Sheikha-Bagheri D, Rogers DWO. Monte Carlo calculation of nine megavoltage photon beam spectra using the BEAM code. Med Phys. 2002;29(3):391-402.

Sicard-Roselli C, Brun E, Gilles M, Baldacchino G, Kelsey C, McQuaid H, Polin C, Wardlow N, Currell F. A new mechanism for hydroxyl radical production in irradiated nanoparticle solutions. Small. 2014;10(16):3338-46.

Strigari L, Ferrero V, Visonà G, Dalmasso F, Gobbato A, Cerello P, Visentin S, Attili A. Targeted dose enhancement in radiotherapy for breast cancer using gold nanoparticles, part 2: a treatment planning study. Med Phys. 2017;44(5):1993-2001.

Tran HN, Karamitros M, Ivanchenko VN, Guatelli S, McKinnon S, Murakami K, Sasaki T, Okada S, Bordage MC, Francis Z, El Bitar Z, Bernal MA, Shin JI, Lee SB, Barberet Ph, Tran TT, Brown JMC, Nhan Hao IV, Incerti S. Geant4 Monte Carlo simulation of absorbed dose and radiolysis yields enhancement from a gold nanoparticle under MeV proton irradiation. Nuclear Instrum Methods Phys Res Sect B. 2016;373:126-39.

Urruticoechea A, Alemany R, Balart J, Villanueva A, Vinals F, Capella G. Recent advances in cancer therapy: an overview. Curr Pharm Des. 2010;16(1):3-10.

Wilkens JJ, Oelfke U. A phenomenological model for the relative biological effectiveness in therapeutic proton beams. Phys Med Biol. 2004;49(13):2811-25. 